FORMATION Formation emploi

Revue française de sciences sociales

122 | Avril-Juin 2013

Relever les défis de la Validation des Acquis de l'Expérience (VAE)

\title{
Le travail des jurys de VAE : le bricolage d'une norme de validation
}

Accreditation of Prior Learning: When jury members build an assessment standard

Die Arbeit der VAE-Prüfungsausschüsse: Fabrizieren einer Norm für die amtliche Anerkennung

El trabajo de los jurados de VAE : el bricolaje de una norma de validación

\section{Stéphane Bellini}

\section{(2) OpenEdition}

Journals

Édition électronique

URL : http://journals.openedition.org/formationemploi/3992

DOI : 10.4000/formationemploi.3992

ISSN : 2107-0946

Éditeur

La Documentation française

Édition imprimée

Date de publication : 30 juin 2013

Pagination : 69-86

ISSN : 0759-6340

Référence électronique

Stéphane Bellini, «Le travail des jurys de VAE : le bricolage d'une norme de validation », Formation emploi [En ligne], 122 | Avril-Juin 2013, mis en ligne le 17 juillet 2013, consulté le 30 octobre 2020. URL : http://journals.openedition.org/formationemploi/3992 ; DOI : https://doi.org/10.4000/ formationemploi.3992

(c) Tous droits réservés 


\title{
Le travail des jurys de VAE : le bricolage d'une norme de validation
}

\author{
StÉPHANe BELLINI \\ Maître de conférences en sciences de gestion \\ Centre de Recherche en Gestion (CE.RE.GE) - université de Poitiers
}

Résumé

Le travail des jurys de VAE : le bricolage d'une norme de validation

Les jurys de VAE sont confrontés à trois problèmes fondamentaux. D'abord, il s'agit pour eux d'identifier l'objet " acquis de l'expérience professionnelle ". Il leur faut ensuite comparer des savoirs différents, entre ceux acquis au cours d'une carrière et ceux acquis sur les bancs de l'Université. Enfin, il s'agit d'évaluer et de décider. Notre recherche montre qu'une telle entreprise n'est possible que par le bricolage d'une norme d'évaluation, permettant aux jurys de se construire des points de référence.

Mots clés : validation des acquis

Abstract

Accreditation of Prior Learning (APL) when jury members build an assessment standard

Accreditation of Prior Learning (APL) mechanism officially recognises, according to a French 2002 law, that work is an instance of acquisition of competence and training. In charge of APL, juries are faced with three main difficulties. First of all, they have to identify what is "professional experience achievements". Second, they have to compare uncomparable forms of knowledge, between those which have been acquired during a professional career and those acquired during courses at University. Third, they have to take a decision. This research reveals the existence of an assessment standard, which enables jury members to take a decision, based on constructed reference points.

Key words: validation of acquired skills

Journal of Economic Literature: I 29

Traduction : Auteur 
La Validation des Acquis de l'Expérience (VAE) est à présent entrée dans les mœurs universitaires avec un rythme annuel de 4100 décisions de VAE dans l'enseignement supérieur (RERS, 2012, p. 276). En reconnaissant officiellement le travail comme vecteur d'apprentissages, la loi de 2002 sur la VAE est présentée comme une seconde chance et un enjeu dans la lutte contre le chômage dans une économie où la mobilité professionnelle devient un impératif.

Mais qu'en est-il de la traduction des intentions de ses concepteurs dans les faits ? Comment les jurys s'emparent-ils de cette question dans leur travail concret d'évaluation des dossiers, quand le décret n $2002-590$ du 24 avril 2002 leur laisse le champ libre sur la méthode, indiquant simplement que le candidat doit expliciter " en référence au diplôme postulé, les connaissances, compétences, aptitudes qu'il a acquises par l'expérience. " ?

En fait, chaque jury est confronté à la difficulté d'un exercice pour lequel il n'a pas été préparé. Au-delà d'un exercice strictement rationnel, le propos de cet article est d'analyser le processus de bricolage d'une norme de validation auquel se livrent les jurys en réponse aux difficultés auxquelles ils sont confrontés.

Nous exposons, d'abord, la complexité de la notion d'expérience professionnelle puis nous montrons que le principe même de la VAE consiste à mettre en correspondance de savoirs incomparables. Nous mettons enfin à jour l'existence d'une norme informelle de validation, par laquelle les jurys parviennent à prendre une décision. 


\section{Encadré 1}

\section{Méthodologie}

Les observations portent sur des jurys de VAE (validation des acquis de l'expérience) de notre IUT (Institut universitaire de technologie), rassemblant des formations des secteurs secondaire (chimie, génie thermique, génie mécanique et productique...) et tertiaire (gestion, commerce, hygiène et sécurité...), dont le point commun est d'avoir un caractère professionnel affirmé ; les résultats de nos observations méritent d'être relativisés en fonction de la méthodologie retenue. L'étude des jurys de formations plus générales pourrait aboutir à d'autres constats.

\section{Matériau empirique}

Nos observations ont porté sur l'examen de 31 candidatures à la VAE, réparties comme suit :

Diplôme universitaire de technologie (DUT) «Techniques de commercialisation » : 2

DUT « Hygiène sécurité et environnement » : 5

DUT « Génie mécanique et productique » : 1

Licence professionnelle (LP) «Animateur Qualité » : 7

$L P$ « Banque-Assurance » 7

LP « Management des échanges internationaux» $: 1$

$\mathrm{LP}$ « Protection civile et sécurité des personnes » : 7

$\mathrm{LP}$ « Valorisation des énergies renouvelables et des technologies de l'environnement » : 1

Nous n'avons procédé à aucune sélection. Les 31 candidatures représentent les demandes de VAE étalées sur l'année universitaire 2005-2006. Nous avons continué à participer à des jurys VAE jusqu'en 2009, cette fois sans enregistrer les données et à des fins de confirmation du bien-fondé de nos analyses.

Nous estimons avoir atteint le seuil de saturation de l'information, seuil à partir duquel une prise de note supplémentaire ne nous apporte plus d'information supplémentaire.

L'ensemble des jurys concerne des diplômes techniques avec référentiel, certains étant nationaux (les DUT), les autres étant locaux (licences professionnelles).

\section{Constitution des jurys}

Au sein de l'université, la composition des jurys est organisée par les composantes pédagogiques (facultés, instituts, ou écoles). Chaque jury est composé de deux membres permanents (président et vice-président), assistant à l'ensemble des jurys de toutes les formations de l'IUT, de deux enseignants-chercheurs du département et deux représentants de la profession. Ainsi constitués, les jurys se sentent légitimes. Ils n'ont pas participé, de près ou de loin, à la constitution du dossier, attribut du conseiller en charge des dossiers relatifs à ces formations. Ce dernier assiste aux jurys sans prendre part ni aux débats ni aux décisions. Son rôle est d'apporter des informations si nécessaire sur des points du dossier (nature du diplôme, éléments de contexte). Dans les faits, il est très peu sollicité par les membres du jury. Les enseignants-chercheurs participant aux jurys le font sur la base du volontariat et, quand les volontaires se font rares, sur sollicitation des chefs de départements. Les membres extérieurs des jurys ne sont pas rémunérés. Pour les enseignants-chercheurs, le barème forfaitaire a été fixé à 20 euros par dossier, l'équivalent d'une demi-heure TD (travaux dirigés) par dossier (examen du dossier et participation au jury). Ainsi, il n'y a pas d'enjeu financier derrière la VAE. 
Notre position dans ce jury est celle de vice-président, position que nous occupions avant le démarrage de la recherche. Cette position nous a permis d'observer l'organisation et le déroulemnt des jurys, depuis l'enregistrement de 21 auditions puis la prise de notes pour dix autres auditions, jusqu'à l'analyse thématique du discours des membres du jury.

\section{Les objets du discours}

Pour comprendre les échanges, nous avons pris connaissance des dossiers des candidats, en tant « qu'objet des activités d'interprétation des jurys», pour reprendre la formule de Mayen et Métral (2008) dont la démarche de recherche est similaire à la nôtre, puisqu'ils observent le fonctionnement des premiers jurys de VAE dans l'enseignement supérieur agronomique. Chaque session a ensuite fait l'objet d'enregistrement des paroles brutes, au cours des trois séquences d'un jury : commentaires précédant le passage des candidats, échanges lors de l'audition des candidats, commentaires suivant l'audition et conduisant à une décision. Nous avons ensuite retranscrit les verbatim de chaque jury sur des fiches, puis nous avons procédé à une analyse thématique du discours afin de distinguer a posteriori les objets du discours. En l'absence (ou malgré l'absence ?) de grille préétablie, trois objets ressortent largement. Ils sont systématiquement abordés avant, pendant et après l'entretien ; ils montrent ce qui est important aux yeux des évaluateurs :

les connaissances acquises d'abord à travers la formation initiale puis par les actions de formation professionnelle ;

l'historique des postes occupés ;

la grille de positionnement, établie pour faciliter le travail d'équivalence entre les compétences et le diplôme. Il s'agit tout simplement de la maquette de formation, détaillée discipline par discipline, dans laquelle chaque candidat s'auto-évalue : pour chaque discipline, il indique ce qu'il pense être son niveau de maîtrise : totale, partielle ou domaine non maîtrisé.

D'autres thèmes apparaissent, mais avec une moindre fréquence (projet professionnel, culture de l'entreprise dans laquelle l'expérience a été vécue) et ne sont pas mobilisés au moment de la décision. 
Nous cherchons à comprendre les mécanismes à l'œuvre au sein d'un jury de VAE. Cette question de recherche impose d'une part, une observation fine du processus de validation, au moment où il se produit; d'autre part, une proximité forte avec les acteurs de ce processus. La participation aux jurys de VAE permet les deux à la fois.

Ce dispositif méthodologique ressort de l'observation participante. Ses intérêts sont bien connus : cette méthodologie permet de recueillir des informations en situation et de saisir ainsi les hésitations, les arguments, le raisonnement des acteurs et leurs critères de décisions. L'observation participante nécessite cependant d'éviter les conséquences négatives liées aux difficultés à rendre compatibles les rôles d'acteur et d'observateur, à se distancier des codes culturels dont l'enquêteur est lui-même imprégné et aux interactions entre le chercheur et les phénomènes observés. Notre objectif est donc à la fois de saisir les opportunités offertes par la proximité du terrain et d'en organiser une distance cognitive (rédaction sur le dispositif méthodologique, le déroulement des jurys, communication régulière auprès de communautés scientifiques). Il s'agit de maintenir constamment une certaine "vigilance méthodologique» (Sardan, 1995), posture faite d'attention et de prise de conscience des interactions entre le terrain et l'observateur.

Chemin faisant, nous avons affiné notre question de recherche, suivant une démarche empirico-inductive. À partir des premières observations, nous constatons en effet que chaque jury de VAE présente des particularités, des configurations différentes qui pourraient avoir un impact sur la décision. Ainsi, la personnalité des membres du jury, leurs propres parcours, les interactions entre le jury et le candidat, les règles d'interaction propres à chaque univers professionnel sont autant d'éléments différenciant les jurys les uns des autres. Pourtant, nous nous sommes davantage intéressés aux points communs des jurys qu’à leurs différences. Notre intention est de repérer les invariants au sein de jurys aux pratiques diversifiées, pour finalement mieux comprendre les mécanismes d'évaluation de l'expérience professionnelle. Cet objectif naît de nos premières observations, quand se dessinent progressivement des traits communs à l'ensemble des jurys.

\section{Cette étrange notion d'expérience professionnelle}

La notion d'expérience est couramment utilisée dans le monde du travail et de la formation. Pourtant, elle mérite d'être questionnée. À lire ou entendre les expressions la véhiculant ("une expérience de trois ans », "une expérience réussie », « une expérience du terrain »), on perçoit bien la polysémie de cette notion. On comprend également qu'elle ne peut être assimilée à l'ancienneté car la seule présence physique dans une situation d'activité n'est pas porteuse de savoirs indépendamment du regard qui est porté sur cette situation. Le fait subjectif de l'expérience est ainsi introduit. À y regarder de plus près, l'expérience «se signale par une opacité qui pourrait la faire regarder comme définitivement 
énigmatique" (Diallo et Clot, 2003, p. 203). Nous tentons ici d'y apporter quelques éclairages conceptuels qui nous paraissent essentiels pour saisir la nature de l'objet qui est évalué en session de VAE : l'expérience professionnelle n'est pas une matière objective qui s'exposerait en un déroulé chronologique d'une carrière. C'est un construit social à multiples facettes, produit notamment de la réflexivité du candidat à l'occasion de la VAE. Cela en complique d'autant plus l'appréciation par des membres de jurys que leur connaissance de l'expérience est empirique.

Nous présentons, dans un premier temps, le caractère multidimensionnel de la notion d'expérience professionnelle. Ensuite, nous mettons en évidence que ses acquis sont en partie invisibles, et que les candidats y ont accès au prix d'un travail de réflexivité. .Enfin, nous mettons en lumière que l'expérience est un objet orienté vers un objectif, ici la VAE.

\subsection{Un objet à plusieurs dimensions}

La difficulté à identifier l'objet " expérience professionnelle » tient d'abord à son caractère polymorphe, mis à jour par de nombreux auteurs. Mayen et Mayeux la considèrent comme une "notion ouverte, polysémique, produit, processus et trajectoire tout à la fois" (2003, p. 17). Ce caractère polysémique est un point commun de ses définitions. Ainsi, pour Mallet et Vernières, l'expérience est constituée d'une dimension technique et d'une dimension sociale (1981). La première dimension recouvre la maîtrise des savoir-faire acquis dans le travail alors que la seconde porte sur l'intégration dans le milieu, la maitrise de la symbolique du travail, qu'il s'agisse des réflexes professionnels ou des codes informels de comportement.

On retrouve à nouveau la définition d'un objet à double face chez Aubret et Gilbert (2003). Pour eux, l'expérience " désigne conjointement des pratiques (activités concrètes) et les effets quelles engendrent sur les individus et les groupes sociaux. Les pratiques sont la partie visible de l'expérience, observable et objectivable. En revanche, ce qui résulte de l'expérience peut être aussi bien de l'ordre de la conscience spontanée, du ressenti, que le produit d'une analyse ou d'une interprétation ». Grasser et Rose distinguent également deux composantes à l'expérience : une composante de savoir-faire et une composante de connaissances spécifiques et tacites du processus de production. Ils complètent en décomposant l'expérience en trois éléments : "cognitifs» (savoirs et savoir-faire), «économiques» (intégration des contraintes de coût et d'économie de temps) et «socialisation" (2000, pp. 5-6).

De son côté, Astier définit trois niveaux d'analyse : "la notion d'expérience désigne à la fois un engagement dans l'action professionnelle dont on valorise alors les aspects inédits pour le sujet ("faire une expérience "), le "vécu " comme éprouvé de cette action singulière et de ses effets ("vivre une expérience") et la transformation de soi résultant de cet engagement ("avoir une expérience")" (2004, p. 34). 


\subsection{Des acquis en partie invisibles}

En évaluant des attitudes de la personne vis-à-vis de son travail ("capacité d'analyse et de proposition », « sens du contact », " esprit critique »...), autant que des compétences plus objectivables car immédiatement liées au poste de travail, les membres du jury dévoilent ce qu'ils recherchent dans les acquis de l'expérience. Ils questionnent l'analyse réflexive du candidat et la transition de "savoirs investis dans des situations historiques " (Schwartz, 2004) à des savoirs plus génériques. Implicitement, ils rejoignent la typologie de Malglaive, qui distingue les "savoirs liés", c'est-à-dire attachés à une situation de travail concrète, les "savoirs autonomes", c'est-à-dire émancipés de situations particulières (démarche scientifique par exemple) et les "savoirs ajustés ", produits de l'adaptation de connaissances théoriques aux situations concrètes (2003, pp. 73-74). Pour les jurys, les «savoirs autonomes » sont un élément fondamental d'une formation universitaire. Il paraît alors logique qu'ils la recherchent chez les candidats au moment de valider une demande de diplôme. Cependant, la preuve matérielle des "savoirs autonomes " est impossible. Par preuve, nous entendons les éléments permettant aux jurys de s'assurer de la maitrise de certains savoirs. Il s'agit alors pour les membres du jury de rassembler suffisamment d'indices ou de «traces» (Clot et Prot, 2003, p. 190) de ces savoirs pour pouvoir évaluer comme l'indique les paroles du responsable de formation à propos d'une candidate à une licence professionnelle dans le domaine de la qualité : " elle a une connaissance de la qualité beaucoup plus large que le candidat précédent, avec plus de hauteur."

\subsection{Un objet issu d'une réflexivité}

L'expérience ne se résume pas à une quantité de temps passée à exercer une activité. Elle n'est pas « un produit-joint de l'activité» (Vincens, 2001, p.22). L'expérience résulte du processus de confrontation de l'individu, avec ses qualifications et ses savoirs, à une situation professionnelle (Astier, 2004 ; Schwartz, 2004 ; Vincens, 2001 ; Grasser et Rose, 2000). En fonction de son degré de réflexivité, c'est-à-dire de mise à distance et de questionnement sur son travail, ses procédures et sur les finalités de l'action, l'individu pourra sortir la compétence acquise de la singularité de la situation professionnelle. À cet égard, le travail qui est demandé lors de la VAE requiert réflexivité puisqu'il vise à faire de l'expérience professionnelle des candidats un objet d'analyse (Vergnies, 2004 ; Magnier et Werthe, 2001). C'est une construction sociale produite par la VAE.

Le Haut Comité Education Economie Emploi le reconnaît : "Au-delà de la forme écrite, c'est l'activité réflexive du candidat qui est importante dans l'élaboration du dossier d'expérience, et sa capacité à orienter son contenu en fonction du référentiel visé. " (2004, p. 57), avant de s'interroger sur la difficulté de verbaliser certaines parties de l'expérience, sur l'inégale diffusion de cette faculté et de pointer l'importance de l'accompagnement pour faciliter la mise à distance. 
Si on considère que l'expérience se caractérise précisément par un processus de réflexivité à partir de l'activité, et que cette réflexivité est encouragée par l'exercice même de la VAE, il faut pointer une nouvelle difficulté de l'identification de l'expérience, puisque, fondamentalement, l'expérience se révèle et se construit par l'analyse distanciée de l'activité et par la mise en mots de pratiques parfois complètement incorporées. Plus encore, l'analyse réflexive produit de la connaissance quand elle permet la prise de conscience de gestes et procédures incorporées, quand elle permet de délier les savoirs des situations dans lesquelles ils ont émergé. Fait à la fois objectif (le poste de travail, les gestes et tâches du quotidien, le collectif de travail) et subjectif (le vécu, l'analyse réflexive du travail), l'expérience devient " communicable en raison des transformations qu'elle subit lorsqu'elle devient objet de réflexion pour le sujet, lorsquelle sénonce ou se partage " (Malavielle, 2005, p. 14). Ainsi donc, la VAE consiste à évaluer un objet que le fait même d'évaluer transforme, voire construit.

\subsection{Un objet orienté}

Plus qu'une description objective du travail du candidat, l'exercice de présentation de l'expérience est une opération de valorisation plus ou moins subtile de l'expérience, en fonction des normes intériorisées par les candidats. Comme l'indique Astier, "l'expérience est toujours doublement orientée vers le passé où elle affirme sêtre construite et vers le futur pour lequel elle se propose comme ressource» (2004, p. 39).

Pour le candidat, se pose la question de l'adéquation entre les normes qu'il a intériorisées et les normes effectives des jurys. Il s'agit de "trouver les mots " et d'adopter la bonne "stratégie de formalisation" (Mayen, 2006, p. 27). Pour le jury de VAE, l'enjeu est de démêler ce qui ressort de l'habillage discursif de l'activité et ce qui ressort de l'expérience, d'où la recherche de preuves. En quelque sorte, tout en évaluant la capacité du candidat à tirer des "savoirs autonomes" de ses «savoirs liés" (Malglaive, ibid.), il s'agit de vérifier que les savoirs énoncés ne s'autonomisent pas trop de l'activité qui est censée les avoir fait émerger.

Cette première partie nous a permis d'appréhender la difficulté, voire l'impossibilité d'évaluer cet objet, ce qui s'explique par la définition même de l'expérience : comment évaluer un objet dont les preuves ne peuvent exister intégralement en raison de sa nature (la dimension subjective produit des acquis invisibles ou en tout cas immatériels), dont la construction s'explique en partie par l'évaluation elle-même ? La complexité de la notion d'expérience professionnelle explique en partie les raisons de l'embarras des jurys, que nous allons évoquer ensuite. Une seconde raison a trait à la comparaison entre savoirs de natures profondément différentes que requiert la VAE. 


\section{La mise en correspondance de savoirs incomparables}

La VAE est un acte d'évaluation. À ce titre, elle est liée à un objet, à une instance légitime, à une finalité, à des points de référence et elle suppose l'application d'une méthode (Rivoire, 2004). C'est une évaluation singulière non seulement par son objet et son instance d'évaluation, points abordés précédemment, mais aussi par la méthode, qui aboutit à valider des compétences hors du contexte qui a permis leur émergence. Enfin, ce qui nous intéresse à présent, c'est la singularité liée à la nécessaire production d'un point de référence, commune à toute évaluation. Or savoirs universitaires et savoirs liés à l'expérience professionnelle ne peuvent s'évaluer à l'aune des mêmes critères de référence tant ils sont différents par nature.

\subsection{Des savoirs évalués à l'aune de références différentes}

Dans le système universitaire, les modes d'évaluation, aussi divers soient-ils, aboutissent à une note, qui valide des niveaux de connaissances ou de capacités à les utiliser selon des modalités définies à l'avance et centrées sur le calcul d'une moyenne. Le contenu à valider est organisé par disciplines avant d'être plus tard jugé dans sa globalité au cours d'un jury. La subjectivité de l'évaluation est omniprésente ; cependant, elle est camouflée derrière l'apparente objectivité des notes, et n'est plus questionnée du fait des habitudes de notation. L'enseignant, spécialiste de son domaine, se crée son point de référence d'autant plus facilement que la répétition dans le temps de l'évaluation lui sert aussi à l'étalonner. En matière de VAE, les repères habituels des enseignants tombent ; la déclaration d'un responsable d'une formation en hygiène et sécurité l'illustre : "C'est pas facile de juger un cas comme celui-là. Il est monté à la force des bras. Si on ne juge qu'à partir de la maquette, c'est mort. "Une enseignante tiendra des propos similaires: "Moi, mettre une note dans ma matière, je sais faire. C'est mon métier. Mettre une note sur un rapport de stage, on a nos grilles, on sait ce qu'on attend, ça va. Mais là, je sais pas faire moi, je sais pas faire. Comment on fait?"

Du côté du milieu professionnel, les procédures d'évaluation sont largement répandues dans les organisations privées et publiques. Les points de référence y sont aussi construits sur des substrats objectifs (nature des missions, résultats, évolution par rapport au passé...) souvent condensés dans une grille d'évaluation. À l'image du professionnel d'un jury d'une formation bancaire, beaucoup de représentants des métiers raisonnent en fonction de leurs pratiques : "je trouve quand même que ça va très vite mais j'ai du mal à situer son employeur (...) car elle a eu beaucoup de courtes expériences avec chaque fois des formations mais ils n'acquièrent pas de professionnalisme sils sont tout le temps en formation. Chez nous, on s'arrange pour qu'il y ait un temps de mise en pratique long... En termes de connaissances, je ne mettrais pas le curseur à "maîtrise" mais "en cours d'acquisition"." 
En matière de VAE, l'universitaire et le représentant du monde professionnel perdent leurs points de référence habituels : le candidat livre une présentation des compétences acquises lors d'expériences professionnelles, sans que celles-ci ne correspondent au découpage académique habituel, sans qu'elles ne soient comparables avec un état précédent, sans qu'elles ne soient complètement vérifiables et sans que ses modalités d'acquisition ne soient maîtrisées par les évaluateurs. L'expérience est un "objet d'embarras" (Mayen et Mayeux, 2003, p. 38), quand il faut en comparer les savoirs acquis à d'autres formes de savoirs, issus d'une formation universitaire.

\subsection{Des savoirs de natures différentes}

Entre " savoirs autonomes » et "savoirs liés ", pour reprendre la distinction de Malglaive, ce n'est pas uniquement le lieu de production des savoirs qui diffère ; plus généraux et abstraits d'un côté, plus spécifiques et concrets de l'autre. C'est aussi la nature des savoirs acquis.

Avec Schwartz, nous relevons que les "morceaux de formation " qu'on cherche à mettre en parallèle avec des " morceaux de savoirs investis " (dans des situations historiques) ne sont des éléments " $\mathrm{n} i$ identiques ni comparables" (2004, p. 20). Les savoirs investis sont " des savoirs qui se donnent en adhérence, en capillarité avec la gestion de toutes les situations de travail, elles-mêmes prises dans des trajectoires individuelles et collectives singulières, contrairement aux savoirs académiques, formels, qui eux sont désinvestis». Les conditions d'émergence des deux types de savoirs, les modes d'apprentissage sous-jacents, leurs finalités, leurs usages, leurs évaluations, les collectifs où ils se partagent, leurs impacts sur les trajectoires personnelles et professionnelles, les aptitudes cognitives qu'ils mobilisent sont autant de différences dans la nature de ces savoirs.

Les propos suivants, tenus par le responsable d'une licence professionnelle dans le domaine de la sécurité, reviennent régulièrement dans les propos des jurys : "Ce qu’on peut voir, c’est la fonction quil occupe et la fonction que peut occuper un étudiant de chez nous. Il tient un poste que les nôtres peuvent tenir. (...) Il lui manque certainement des trucs mais bon (...). On regarde si le poste équivaut à un niveau de formation ». Ils montrent que pour parvenir à la mise en correspondance, les jurys observés estiment, comme Labruyère, que «le principe de la VAE repose sur l'hypothèse que l'expérience d'un ou de plusieurs emplois permet, dans certaines conditions, de développer des compétences et des savoirs d'action qui ont la même valeur, en termes de capacité d'action et de potentialité de développement, que les savoirs acquis en formation. On névalue pas la même chose dans les deux situations (formation formelle et expérience), mais on valide la même capacité d'action, le même potentiel de développement " (Vergnies, 2004, p. 8). 


\section{La décision malgré tout}

De la complexité conceptuelle de l'expérience professionnelle on pourrait légitimement conclure que la VAE est un exercice impossible tant l'objet à valider est difficile à saisir. L'anecdote suivante est significative de bien des situations observées : à la fin de l'audition d'un candidat au parcours chaotique pour un DUT du domaine commercial, le candidat sort de la salle. Suivent alors quelques secondes de silence, pendant lesquelles les membres du jury, universitaires et professionnels, se regardent, comme s'ils cherchaient chez les autres des certitudes qu'ils n'avaient pas eux-mêmes. Puis la salle résonne d'un profond soupir collectif, expression sonore du désarroi du jury. Pourtant, même dans le cas présent (VAE partielle), chaque jury s'accorde assez rapidement sur une décision. En quelques échanges, sans vote ni tour de table formel, un consensus émerge pour quasiment tous les dossiers. Le constat est partagé par d'autres recherches du même type (Mayen et Métral, 2008 ; Morisse, 2007). La décision résulte d'échanges d'impressions, d'arguments sur la base d'examen des dossiers et des programmes pédagogiques. Se crée ainsi, dans le non-dit des regards croisés et derrière les mots des membres du jury, une norme d'évaluation par laquelle on reconnaît la valeur d'une expérience, on la jauge ou on la dénie. Elle coexiste avec les normes d'évaluation en cours dans les jurys habituels.

\subsection{La recherche de points de référence}

Pour fonder une décision, les membres du jury examinent les dossiers puis auditionnent les candidats. Ce faisant, ils cherchent à reconstituer des points de référence dont ils sont au départ dépourvus pour finalement étayer un avis ; ce phénomène est également observé par Cherqui-Houot (2006), pour qui les membres de jury ont tendance à ramener les caractéristiques des candidats à la VAE à des "standards " étudiants ; en témoigne la citation suivante, précédant une audition : "Son dossier est un vrai roman. Il a eu des expériences multiples, dans différentes institutions, de l'armée de terre à la direction d'une filiale d'entreprise de transport international. Il a créé une entreprise de conseil... En bref, au-delà des péripéties de sa carrière, il semble avoir une certaine compétence en matière de transport et une expérience variée qui correspond finalement assez bien à ce qu'on veut faire en licence. "

Dans le huis clos de leurs bureaux, les membres du jury de VAE étudient les dossiers des candidats. Ceux-ci dépassent fréquemment la centaine de pages. Y sont souvent consignés une fiche de poste, le compte-rendu de réunions signalant une intervention ou un rôle particulier dans une action, la photocopie des actions de formation passées... Que reste-t-il alors dans l'esprit des membres de jurys de ces dossiers volumineux ? Que retiennent-ils du dossier d'une vie au travail ?

L'entretien est un temps fort d'un jury de VAE. Les questions y sont posées indifféremment par les professionnels du domaine de formation ou par les universitaires ; celles-ci portent sur les trois thèmes cités dans l'encadré méthodologique (formation, historique 
des postes et grille d'auto-positionnement) et sont complétées de questions relatives au projet du candidat. D'autres questions apparaissent plus marginales, sur la politique de l'entreprise par exemple. Au-delà des impressions générales, nous cherchons à mettre à jour ce qui constitue le point de référence en reprenant les trois principaux objets du discours, à savoir le parcours de formation du candidat, l'historique des emplois occupés et la grille d'auto-positionnement.

\subsubsection{La formation : un indicateur fondamental}

Les questions relatives au niveau de formation initiale et aux diplômes révèlent l'importance que les jurys lui accordent et qu'ils en font un point de référence fondamental. C'est un indicateur à la fois de compétences techniques qu’on suppose acquises, compte tenu d'un brevet de formation, mais aussi de qualités plus générales («facultés de réflexion » par exemple).

Quand les jurys commentent les dossiers qu'ils ont examinés avant l'audition des candidats, avec une régularité frôlant le systématisme, ils commencent par parler de la formation initiale ou des manques d'information sur celle-ci, à l'image des premiers propos d'un enseignant au sujet d'un candidat à la VAE pour une formation dans le commerce international : "C'est quelquiun qui n’a pas le bac. Ce serait inédit de délivrer la licence à quelqu’un ayant ce profil. " Ainsi, la décision de validation est plus facilement acceptée quand un candidat a un niveau de qualification proche du diplôme demandé : «Il a déjà un bac +2 , la marche n'est pas trop haute. " (propos d'un enseignant-chercheur). L'inverse se retrouve aussi : "C'est plus difficile de valider la licence quand on vient du CAP (...). Avec un CAP, il lui manque de linstruction générale, un raisonnement. " (un représentant de la profession).

Cet exemple montre qu’à expérience professionnelle jugée équivalente, la présomption d'aptitudes à la réflexion ou de capacités d'analyse n'est pas la même. Nous voyons là un paradoxe : alors que la VAE suppose un examen de l'expérience professionnelle, une prime est donnée à la formation initiale comme mode de production essentiel de certaines démarches intellectuelles ou de façons de travailler. Le diplôme est perçu comme un signal de compétences.

On assiste ici à un glissement de l'évaluation : de l'évaluation de l'expérience à l'évaluation de qualités censées être développées en centre de formation, sans que cela ne soit vérifié par ailleurs. Ce glissement résulte avant tout de l'embarras des membres du jury face à l'exercice de la validation.

\subsubsection{L'historique des emplois occupés}

L'historique des postes occupés constitue un élément important servant de point de référence aux évaluateurs. Le jury évalue les compétences produites par un parcours professionnel. Comme le soulignent Mayen et Métral, il examine «l'expérience processus» à partir de "l'expérience trajectoire " (2008, p. 190). 
Cependant, le processus de validation d'une expérience est facilité quand l'identification nette d'un emploi, d'un statut ou d'une progression de carrière fournit un point de référence clair pour le jury. D'un capitaine des sapeurs-pompiers de Paris, le responsable de formation peut affirmer avant de le recevoir qu' " on peut valider les yeux fermés " compte tenu de son poste (validation d'une licence professionnelle "Protection Civile et Sécurité des Personnes»). Le représentant de la profession renchérit : "Il tient la route. Pour être capitaine aux pompiers de Paris, il faut avoir un sacré niveau. "Dans ce cas, la décision d'une VAE totale est prise en dix secondes. En revanche, quand le point de référence est moins clair pour les jurys, la situation est plus embarrassante. La citation suivante l'illustre. Il s'agit d'une validation pour un DUT en "Techniques de commercialisation ». Le responsable de la formation prend la parole : "Ce qui me gêne pour la validation totale, bon, il a 39 ans, bon, même sil est intéressant, il y a, bon c'est pas de sa faute, il y a peu d'autonomie. Si on considère nos étudiants en sortie de DUT, à 39 ans, on peut espérer quils auront plus d'autonomie."

\subsubsection{La grille d'auto-positionnement pour réguler l'activité de validation}

Avec cette grille (présentée plus haut), on retourne la charge de l'évaluation. C'est au candidat de s'évaluer et d'apporter des éléments de preuve de ses affirmations. À partir de ce document, le travail du jury consiste à évaluer cette auto-évaluation et à en vérifier la crédibilité, à l'image de cet avis formulé avant l'audition par la représentante d'une formation bancaire : "Elle s'auto-évalue dans tous les domaines, c'est trop. Il faut l'interroger là-dessus ."

La grille d'auto-positionnement facilite une démarche de validation dite "terme à terme ", qui consiste à morceler les compétences acquises par le candidat et à les traduire en disciplines académiques. Pour les jurys se livrant à un tel exercice, la manœuvre permet de basculer d'un terrain inconnu (l'expérience) à un domaine maitrisé (le référentiel), d'un objet à évaluer sans point de référence, au traitement du même objet dans une grille connue. Via la grille d'auto-positionnement, le référentiel de formation est un point de référence central dans la décision des jurys; c'est un «instrument qui régule l'activité de validation en orientant le travail des jurys vers un document» (Prot, 2003, p. 227).

Cependant, l'exercice ne préserve pas de la subjectivité pourtant fuie quand il s'agit d'intégrer à la décision les principes de compensation régissant l'acquisition d'un diplôme. Dans les faits, l'expérience professionnelle ne se fond jamais complètement dans le moule d'une maquette de formation. Les deux citations suivantes le montrent bien. Elles sont formulées par le même membre du jury, enseignant en licence professionnelle, et concernent le même dossier, avant et après l'audition :

Avant l'audition : "En fait, pour me faciliter la tâche lors des jurys de VAE, j'évalue par rapport aux différentes UE [unités d'enseignement]. J'ai une grille qui reprend les matières et les UE et j'évalue si c'est bon ou pas. Dans son cas, pour tout ce qui est professionnel, pas de problème. Je suis moins convaincu par ce qui est plus académique "; 
Après l'audition : "Si je regarde le programme, bon, la création de site internet, il ne sait probablement pas faire mais c'est un problème de détail. On ne va pas chipoter là-dessus. Bon, pour l'économie internationale, c'est pareil... " Résultat: VAE totale.

\subsection{L'expression d'une norme de validation}

Le travail de validation des jurys se fonde sur des éléments formalisés (formation, postes de travail, grille d'auto-positionnement) qui ne prennent toutefois leur sens qu'à l'aune d'une norme implicite, sorte de toise cognitive aux graduations non dites, dont les contenus peuvent varier. Morisse (2007, pp. 4-5) montre qu'au sein de jurys du domaine agricole (Bac et BTS - brevet de technicien supérieur), les critères d'évaluation non dits et " allant de soi " concernent la façon d'être au travail (courageux, autonome, motivé), la façon d'être avec les autres (franc, honnête, spontané) et un genre de discours (à l'aise, concret). De son côté, Werthe (2003, p. 263) constate une "activité à double fond " chez un jury de VAE de BTS «Assistant de direction ", qui consiste à se fonder sur la qualité de synthèse et les fautes d'orthographe du dossier pour prendre une décision. Habitués à évaluer des productions écrites, les membres du jury peuvent, par cette méta-analyse informelle, évaluer les candidats en les comparant à une norme. Mayen et Métral (2008) observent que les évaluations des jurys s'appuient sur un « ressenti ", fondé sur les "indicateurs de capacités en référence à des univers de travail». Il ne s'agit pas d'un ressenti «sauvage» (p. 188). De notre côté, nous montrons que les jurys reconstituent des points de repère connus pour évaluer un objet méconnu.

Ces observations concourent à révéler le bricolage d'une norme, dont la fonction, bien qu'informelle, est de donner des repères, d'installer rapidement des routines dont on ne questionne plus le bien-fondé et finalement de rendre possible une décision. En ce sens, elle a des vertus opérationnelles. Ce qui est étalonné à la norme n'est ensuite jamais l'expérience professionnelle en tant que telle mais la partie de ses acquis qui sont mis en scène dans un artefact nommé « dossier de validation ».

La prouesse du décalque acquis de l'expérience/acquis de la formation n'est alors possible que par une méta-analyse informelle du discours ou de l'écrit, selon laquelle l'exercice de la transmission véhicule autant de significations que son contenu; les jurys cherchent dans les façons de parler et les mots employés des éléments pour les étalonner à la norme. En effet, certaines dispositions sont jugées essentielles par les membres des jurys, comme la capacité à prendre du recul, à diagnostiquer, à proposer, à travailler en autonomie mais elles ne font l'objet d'aucune évaluation explicitée. On retrouve ce raisonnement pour la plupart des diplômes. Un représentant du milieu bancaire déclare par exemple à propos d'un candidat à une licence professionnelle " banque-assurances " qu' « il a une vision globale, il connaît son sujet».

Sans formaliser leur processus d'évaluation, les membres des jurys font porter leurs questions sur les «savoirs liés", acquis dans les situations concrètes de travail et leur attribuent 
implicitement des significations sur les "savoirs autonomes " sans les questionner directement (Malglaive, ibid.). À cet égard, la capacité d'un candidat à argumenter, à généraliser, à analyser des situations et, plus généralement, tout ce qui le fait sortir d'un rôle d'exécution est valorisé. Cela signifie que la dimension technique de l'expérience ne suffit pas à valider un diplôme.

Ainsi, c'est par la succession de mécanismes informels que l'exercice de la VAE devient possible : réduction de la complexité de l'objet "expérience professionnelle ", recours à une norme d'évaluation et à une méta-analyse du dossier et/ou du discours.

\subsection{Un déploiement de la norme facilité par une proximité socioculturelle}

La constitution du jury apporte un éclairage sur le mode de décision. En effet, la norme informelle se déploie d'autant plus aisément que les membres des jurys témoignent d'une certaine proximité socioculturelle, même s'ils appartiennent à des univers professionnels différents. Ils rassemblent trois caractéristiques communes : ils sont volontaires, ont un vocabulaire partagé et sont attachés aux diplômes.

Tout d'abord, la participation (étude du dossier, participation au jury) se fait sur la base du volontariat. Notons qu'il peut y avoir, au sein des formations, des universitaires opposés à la VAE, jugeant qu'on dévalorise le diplôme à le décerner sans donner la formation. Ces opposants ne participent pas aux jurys, soit parce qu'ils s'y refusent soit parce qu'ils n'y sont pas invités.

Ensuite, le vocabulaire des professionnels et des universitaires est largement partagé. Les professionnels et universitaires posent des questions et échangent sur les mêmes thèmes et avec les mêmes mots. Ce phénomène a trois principales explications. D'abord, les universitaires présents dans les jurys sont fortement impliqués dans leurs formations et sont en lien étroit avec les partenaires professionnels du secteur. Ils ont une bonne connaissance des métiers auxquels ils sont censés préparer leurs étudiants. Ensuite, les professionnels présents ont eux-mêmes une bonne connaissance des formations, soit parce qu'ils en sont issus, soit parce qu'ils en sont des intervenants ou qu'ils participent à des jurys (recrutement, délivrance de diplôme). Enfin, la constitution des jurys revient aux responsables des formations, qui mobilisent dans leurs réseaux les personnes les plus à même de participer aux jurys de VAE, par leurs connaissances, leurs engagements ou leur sensibilité.

Enfin, on trouve dans les parcours des membres des jurys des emplois d'enseignants du supérieur et de professionnels titulaires d'un diplôme au moins égal à celui qu'ils vont délivrer, c'est-à-dire une population diplômée de l'enseignement supérieur et qui attribue aux diplômes une grande valeur. 
Ainsi constitué, le jury met au point une règle du jeu informelle dans son activité. À cette aune, la VAE peut être considérée comme un champ d'application supplémentaire du principe de régulation autonome (Reynaud, 1989), c'est-à-dire de règle du jeu établie par les parties-prenantes d'une activité, en autonomie de la règle prescrite, chacun trouvant dans les règles informelles un cadre à l'action plus confortable, plus pertinent ou plus apte à ménager les intérêts de chacun. En l'occurrence, la construction de la règle du jeu peut être interprétée non comme un moyen de se détacher des prescriptions organisationnelles mais comme une manière de créer des repères et de se constituer un cadre à l'action. En organisant des jurys dont les membres ont un certain nombre de points communs, on constitue une sphère sociale familière, qui facilite ellemême l'émergence de la règle du jeu.

\section{Conclusion}

L'expérience professionnelle est un objet bien plus complexe à identifier que le langage courant ne le laisse croire : il mêle des dimensions objective et subjective, dont les preuves matérielles sont en partie inexistantes, et il s'agit d'un objet construit par le regard réflexif. De cette manière, la VAE fait bien plus que valider l'expérience ; elle prend part à sa construction.

De plus, l'exercice vise à comparer les savoirs acquis en formation et ceux acquis dans le travail alors que, par nature, ces savoirs sont différents. L'exercice exigé des jurys peut ainsi paraître paradoxal. Les jurys le rendent pourtant possible en réduisant la complexité à la fois de l'objet à valider et de la comparaison à réaliser. Ils bricolent une norme informelle d'évaluation; elle sert à étalonner les dossiers et à formuler un avis.

Le travail présenté interroge au premier chef les pratiques d'évaluation des jurys de VAE et l'existence de possibles alternatives aux solutions bricolées par les jurys ; un des problèmes étant celui de l'équité entre jurys dont les normes sont différentes puisque construites localement, sans harmonisation ni concertation avec d'autres jurys. Nous pensons que le chantier mérite en tout cas d'être ouvert et que c'est en connaissant mieux ses propres façons de fonctionner qu'un jury peut faire évoluer ses pratiques.

Plus encore, un prolongement de ce travail sur les effets de la participation des enseignants-chercheurs aux jurys de VAE sur leurs conceptions de la formation mériterait d'être entrepris. En effet, si les savoirs autonomes sont un critère de l'évaluation lors de la VAE, n'est-il pas important de les développer en formation, en explicitant les moments ou lieux où ils sont produits? Nous rejoignons Prot pour qui l'activité de VAE ne cesse d'avoir pour toile de fond l'activité habituelle des membres des jurys. "Valider les acquis peut ainsi devenir un moyen de développement" des formations et des formateurs, "une ressource pour faire son travail et non une tâche supplémentaire" (Prot, 2004, p. 123). 


\section{- Bibliographie}

Astier P. (2004), "Parler d'expérience ", Formation Emploi, n 88, octobre-décembre, pp 33-42.

Aubret J., Gilbert P. (2003), Valorisation et validation de l'expérience professionnelle, Dunod, Topos, Paris.

Cherqui-Houot I. (2006), "VAE : les universités à l'épreuve de l'expérience ", Savoirs, Revue internationale de recherches en éducation et formation des adultes, ${ }^{\circ} 10$, pp. 93-110.

Clot Y., Prot, B. (2003), «Expérience et diplôme. Une discordance créatrice ", L'orientation scolaire et professionnelle, 32, $\mathrm{n}^{\circ}$ 2, pp. 183-201.

Diallo M. D., Clot Y. (2003), «L'exploration de l'expérience dans l'analyse de l'activité : problèmes de méthode ", L'orientation scolaire et professionnelle, 32, n 2, pp. 203-217.

Grasser B., Rose J. (2000), « L'expérience professionnelle, son acquisition et ses liens à la formation ", Formation Emploi, n 71, juillet-septembre, pp. 5-19.

Haut Comité Education Economie Emploi (2004), VAE : construire une professionnalisation durable. Pourquoi un rapport d'étape ?, Paris, La Documentation française.

Magnier J., Werthe C. (2001), «L'expérience revisitée à l'occasion de la validation des acquis professionnels », Formation Emploi, n 75 , pp. 29-41.

Malavielle L. (2005), Accompagnement des professionnels en vue de la VAE. Vers une nouvelle forme de formation continue, Mémoire du DESS Conseil et formation en éducation, Montpellier.

Malglaive G. en débat avec Aubret J. (2003), « La validation des acquis de l'expérience », Savoirs, $\mathrm{n}^{\circ} 1$, pp. 57-82.

Mallet L., Vernières M. (1981), «L'expérience professionnelle dans les modes de gestion de la main-d'œuvre ", Travail et emploi n ${ }^{\circ}$ 9, juillet-septembre, pp. 65-74.

Mayen P., Métral J.-F. (2008), "Compétences et validation des acquis de l'expérience ", Formation Emploi, nº 101, pp. 183-196.

Mayen P. (2006), "Evaluer avec l'expérience », in Figari G., Mottier Lopez L., Recherche sur l'évaluation en éducation, Paris, L'Harmattan, coll. « Evaluer », pp. 25- 33.

Mayen P., Mayeux C. (2003), «Expérience et formation », Savoirs, nº 1, pp. 15-53.

Morisse M. (2007), "Analyse de l'activité évaluative des membres de jury de VAE », Actes du 19e colloque de l'Admée - Europe, 2007, vol. 1.

Prot B. (2003), "Analyse du travail des jurys en validation des acquis : l'usage des référentiels », L'orientation scolaire et professionnelle, 32, n 2, pp. 219-243. 
Prot B. (2004), " Le doute, le concept et le collectif. Trois ressources pour le développement de l'activité d'un jury ", Education permanente n ${ }^{\circ}$ 158/ 2004-1, pp. 115-126.

Repères et Références Statistiques sur les enseignements, la formation et la recherche - RERS (2012), publication du ministère de l'Enseignement Supérieur et de la Recherche.

Reynaud J.-D. (1989), Les règles du jeu. L'action collective et la régulation sociale, Armand Colin, Paris.

Rivoire B. (2004), "VAE : par quel détour passe l'évaluation ", Education permanente, $\mathrm{n}^{\circ}$ 159/2004-2, pp. 79-90.

Sardan de O. (1995), « La politique de terrain. Sur la production de données en anthropologie ", Enquête, 1, pp. 71-109.

Schwartz Y. (2004), "L'expérience est-elle formatrice ? ", Education permanente, n $158 /$ 2004-1.

Vergnies J.-F. (2004), « La validation des acquis de l'expérience, nouveaux enjeux, nouveaux débats ", Entretien avec Chantal Labruyère et José Rose, Formation Emploi, n 88.

Vincens J. (2001), « Définir l'expérience professionnelle », Travail et Emploi ${ }^{\circ}{ }^{85}$, janvier, pp. 21-34.

Werthe C. (2003), «L'évaluation collective : l'exemple d'un jury de validation des acquis ", Lorientation scolaire et professionnelle, 32, $\mathrm{n}^{\circ} 2$, pp. 245-268. 